\section{Intralobar pulmonary sequestration and mediastinal bronchogenic cyst}

\author{
Ritu G Grewal, Chun K Yip
}

\begin{abstract}
A patient with a bronchogenic cyst and intralobar pulmonary sequestration is presented. Chest radiography showed a posterior mediastinal mass and a computed tomographic scan of the chest revealed, in addition, an abnormality suggestive of pulmonary sequestration. This was confirmed by an aortogram. It is important to be aware of the coexistence of these anomalies to make a diagnosis preoperatively.
\end{abstract}

(Thorax 1994;49:615-616)

Developmental anomalies of the lung are rare. They span a continuum of maldevelopment involving the pulmonary parenchyma, the pulmonary vessels, or both. ${ }^{1}$ Bronchogenic cysts result from abnormal budding of the primitive tracheobronchial tree. Pulmonary sequestration is a cystic mass of non-functioning lung tissue that has an anomalous systemic blood supply. ${ }^{1}$ A review of the literature showed only one previous report of intralobar pulmonary sequestration and bronchogenic cyst in the same patient. $^{2}$

\section{Case report}

A 36 year old white woman was admitted for evaluation of an abnormal chest radiograph. She had been treated for a left lower lobe pneumonia three months before admission. Past history was significant for frequent respiratory infections for more than 10 years. Physical examination was unremarkable. Routine laboratory examination and arterial blood gas tensions were normal. Pulmonary function tests showed a mild restrictive ventilatory defect. Chest radiography revealed a left paraspinal, posterior mediastinal mass and an opacity in the posteromedial basilar area of the left lung (fig $1 \mathrm{~A}$ and $\mathrm{B}$ ). A computed tomographic (CT) scan of the chest did not show communication between the posterior mediastinal mass and the spinal column. In addition, an increased density suggesting pulmonary sequestration was seen in the left lower lobe. An aortogram showed the blood supply to the density arising from the descending aorta apparently below the diaphragm, confirming the diagnosis of pulmonary sequestration (fig 2). Fibreoptic bronchoscopy revealed a normal
A

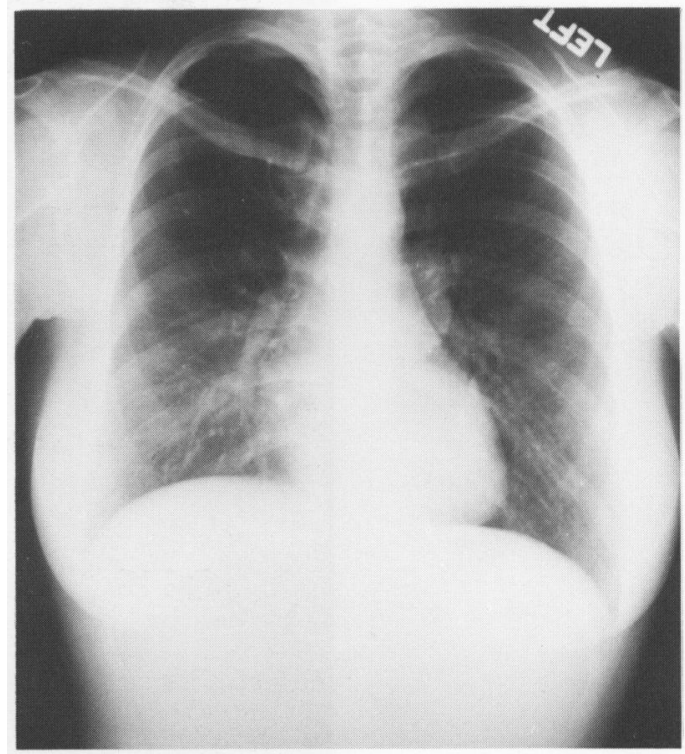

B

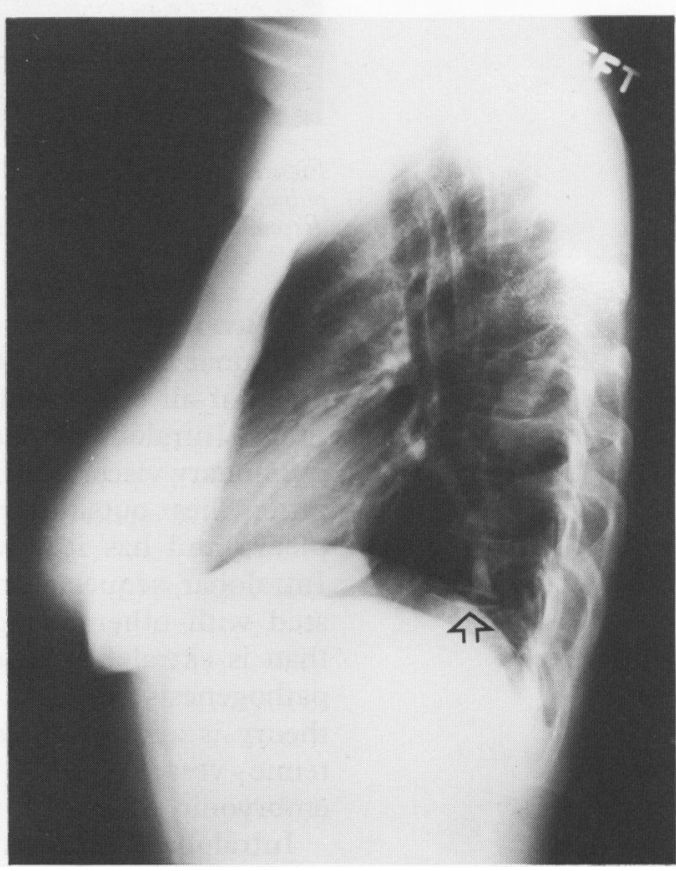

Figure 1 Posteroanterior $(A)$ and lateral $(B)$ views of the chest radiograph showing a mediastinal mass. An opacity in the posteromedial basilar area (arrow) is also seen in the lateral view.

bronchial anatomy. A left bronchogram showed anterior displacement of a normal bronchial tree and absence of communication with the sequestration. Barium swallow showed no communication of the pulmonary sequestration with the gastrointestinal tract. Magnetic resonance imaging (MRI) of the spine revealed that the paraspinal mass was not arising from the spinal cord. The patient underwent a left lower lobectomy and resection of the posterior mediastinal mass. This was a bronchogenic cyst filled with dark green fluid. The abnormal density in the left lower lobe was an intralobar pulmonary sequestration. However, the artery to the sequestration was found to be arising from the aorta above the diaphragm. 


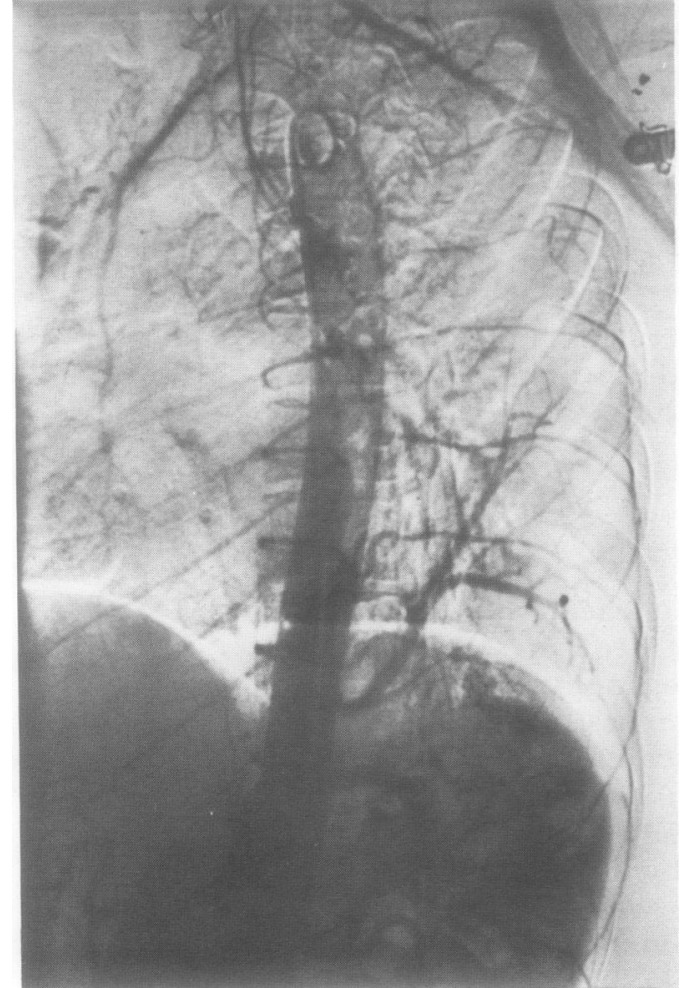

Figure 2 Aortogram showing the blood supply to the pulmonary sequestration appearing to arise from the descending aorta below the diaphragm.

\section{Discussion}

Pulmonary sequestration comprises $0 \cdot 15-$ $6.4 \%$ of all congenital pulmonary malformations. ${ }^{3}$ Intralobar sequestration lies within the pulmonary visceral pleura; extralobar sequestration lies outside the pulmonary visceral pleura and has its own investing envelope. ${ }^{3}$ Intralobar sequestration is less often associated with other congenital anomalies (14\%) than is extralobar sequestration $(50 \%) .{ }^{4}$ The pathogenesis is widely debated; the prevailing theory is a failure of obliteration of the systemic vessels which arise from the early embryonic splanchnic vessels. ${ }^{5}$

Intralobar sequestration is most commonly found in the posterior basal segment of the lower lobes. ${ }^{4}$ The aberrant artery originates from the aorta in approximately $75 \%$ of cases. The venous return is usually via the pulmonary veins. ${ }^{4}$ Intralobar sequestration should be resected because of the propensity for recurrent bouts of infection in the surrounding compressed lobe. As a result of tissue necrosis due to infection, a communication between this sequestration and the bronchial tree may form. This can also result in repeated infections. ${ }^{6}$ The finding of a shadow that fails to clear radiographically, especially in the left lung base as occurred in this patient, should elicit suspicion and be investigated with CT scanning or MRI.

Pulmonary sequestration has a variable appearance on the CT scan. It may be solid, cystic, or contain air. If the anomalous aortic branches supplying the sequestration are large they may be visible on the CT scan arising from the aorta at or near the same level as the lung lesion. If intravenous contrast medium is injected the sequestration or segments of its wall may opacify at the same time as the descending aorta. ${ }^{7}$

The diagnosis of pulmonary sequestration must be confirmed by an aortogram before surgery to prevent uncontrolled haemorrhage from inadvertent injury to a systemic artery during resection. ${ }^{5} M R I$ and $M R$ angiography have been shown to be valuable in defining the arterial and venous supply of the sequestered and normal lung and may replace aortography. ${ }^{89}$ All patients should undergo an upper gastrointestinal series to identify communication with the gastrointestinal tract. ${ }^{6}$

Bronchogenic cysts may be mediastinal or intrapulmonary. The lower lobes are commonly involved. ${ }^{5}$ An intrapulmonary bronchogenic cyst results from an earlier embryological insult than does a mediastinal cyst. ${ }^{1}$ We found only one report where a bronchogenic cyst was associated with intralobar pulmonary sequestration. $^{2}$ It is unclear why these two developmental anomalies of the lung are not associated more frequently, as the timing of origin for both is felt to be in the sixth to eighth week of embryological development. ${ }^{1}$

With the increased use of CT scanning and MRI these anomalies may be diagnosed more frequently. It is important to be aware that they can coexist, and any suspicion should lead to an appropriate preoperative evaluation to prevent surgical morbidity.

1 Panicek DM, Heitzman ER, Randall PA, Groskin SA, Chew FS, Lane EJ, et al. The continuum of pulmonary developmental anomalies. Radiographics 1987;7:747-72.

2 Croyle P, Estrera AS. Bronchogenic cyst and intralobar sequestration mimicking thoracic aortic aneurysm. South Med ₹ 1982;75:1267-8.

3 Sade RM, Clouse M, Ellis FH. The spectrum of pulmonary sequestration. Ann Thorac Surg 1974;18:644-58.

4 Savic B, Birtel FJ, Tholen W, Funke HD, Knoche R. Lung sequestration: report of seven cases and review of 540 pequestration: report of seven cases and

5 Luck SR, Reynolds M, Raffensperger JG. Congenital bronchopulmonary malformations. Curr Probl Surg chopulmonary

6 Shamji FM, Sachs HJ, Perkins DG. Cystic disease of the lungs. Surg Clin North Am 1988;68:581-620.

Webb WB. CT of solitary pulmonary vascular lesions. Semin Roentgenol 1984;29:189-98.

8 Pessar ML, Soulen RL, Kan JS, Kadir S, Zerhouni EA MRI demonstration of pulmonary sequestration. Pediatr Radiol 1988;18:229-31.

9 Doyle AJ. Demonstration of blood supply to pulmonary sequestration by MR angiography. $A \mathcal{F}$ 1992;158:989-90. 